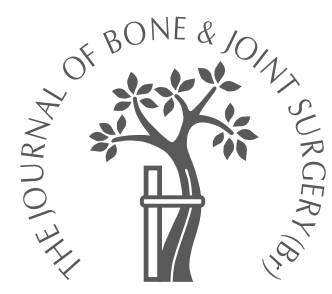

\title{
Retrieval of a well-functioning extensor mechanism allograft from a total knee arthroplasty
}

\author{
CLINICAL AND HISTOLOGICAL FINDINGS
}
R. S. J. Burnett, V. L. Fornasier,
C. M. Haydon,
B. M. Wehrli,
C. N. Whitewood,
R. B. Bourne

From the University of Western Ontario, London, Canada
In. S. J. Burnett, FRCSC, Clinical Fellow C. M. Haydon, HBS, Research Fellow B. M. Wehrli, FRCPC Lecturer

C. N. Whitewood, FRACS, Clinical Fellow

R. B. Bourne, FRCSC, Professor, Chairman London Health Sciences Center, 339 Windemere Road, 9th Floor 90F-2, University of Western Ontario, London, Ontario N6A 5A5, Canada.

V. L. Fornasier FRCPC, Professor, Director of Laboratory of Bone and Joint Pathology

University of Toronto, 30 Bond Street, Toronto, Ontario M5B 1W8, Canada

Correspondence should be sent to Mr R. S. J. Burnett at Department of Orthopedic

Surgery, Washington

University School of

Medicine, Campus Box 8233, 660 South Euclid Avenue, St Louis, Missouri 63110, USA.

(C)2004 British Editorial Society of Bone and Joint Surgery doi:10.1302/0301-620X.86B7 $15182 \$ 2.00$

$J$ Bone Joint Surg $[\mathrm{Br}]$ 2004;86-B:986-90.

Received 2 December 2003;

Accepted after revision

15 April 2004

\begin{abstract}
We present the histological findings of an extensor mechanism allograft which was used in a total knee arthroplasty two years after implantation. Analysis of the graft was undertaken at four distinct anatomical levels and it was found to be incorporated into host tissue at each level. A wedge of fibrinoid necrosis, probably related to impingement of the graft on the tibial polyethylene insert, was seen. Impingement may play a role in the injury and necrosis of an allograft and may be one mode of failure in an extensor mechanism allograft.
\end{abstract}

Failure of the extensor mechanism after a total knee arthroplasty (TKA) is a devastating complication and may occur in up to $2.5 \%$ of patients. ${ }^{1,2}$ The use of an extensor mechanism allograft to reconstruct the deficient or ruptured extensor mechanism of the knee after TKA has been reported previously. ${ }^{3-10}$ Early results have been encouraging although a high complication rate $e^{4,5,7,8}$ with allograft failure, attenuation, and clinical extensor lag have been identified.

To our knowledge, this is the first study to report the retrieval of a well-functioning extensor mechanism allograft after a TKA. The purpose of this study was to report the clinical and histological findings at a minimum of two years after primary TKA, with reconstruction of the extensor mechanism, using a tendo Achillis bone block allograft. The patient provided informed consent for the use of her clinical, histological and radiographic findings for this study.

\section{Case Report}

A 49-year-old woman was referred in January 2000 with a history of having undergone a patellectomy and subsequently an arthrodesis of the right knee in 1985. She had developed chronic right hip, back, and some right knee pain despite a solid arthrodesis. These symptoms led her to seek a referral for consideration of conversion of the arthrodesis to a TKA rather than an above-knee amputation.

Pre-operative physical examination revealed a healthy Caucasian woman whose weight was 110 pounds and whose height was $157.5 \mathrm{~cm}$. She walked with a short-leg $(2 \mathrm{~cm})$, circumduction pattern on the right. Examination of the right knee showed two longitudinal scars, one medial and one lateral. The knee had been fused in full extension neutral varus-valgus and neutral rotation. She was able to actively straight leg raise. The quadriceps musculature was significantly wasted, although it was possible to elicit a palpable contraction proximally. Neurological and vascular examination was normal and plain radiographs showed a sound arthrodesis. MRI showed an extensively scarred extensor mechanism but a preserved proximal quadriceps muscle group. An erythrocyte sedimentation rate (ESR) and C-reactive protein (CRP) level were $5 \mathrm{~mm} /$ hour and 0.5 , respectively.

The options discussed included no surgery, above-knee amputation and conversion of the arthrodesis to a TKA. The need for an extensor mechanism allograft in order to reconstruct the deficient and scarred extensor mechanism was also discussed. The patient was told of the high complication rates associated with the conversion of an arthrodesis to a TKA ${ }^{11-14}$ but she wished to proceed. This was undertaken in April 2000 and required an extensor mechanism allograft to reconstruct the deficient extensor mechanism. The knee was prepared in a standard fashion using revision instrumentation (Genesis II Revision Knee, Smith and Nephew, Memphis, Tennessee) trial implants with stem extensions. The range of movement with the trial implants in place before inserting the allograft was $0^{\circ}$ to $120^{\circ}$. Preparation and insertion of the tendo Achillis and calcaneal bone block allograft was performed as described by Crossett et al. ${ }^{10}$ The tendo Achillis portion was split proximally and longitudinally into two arms and weaved through the 


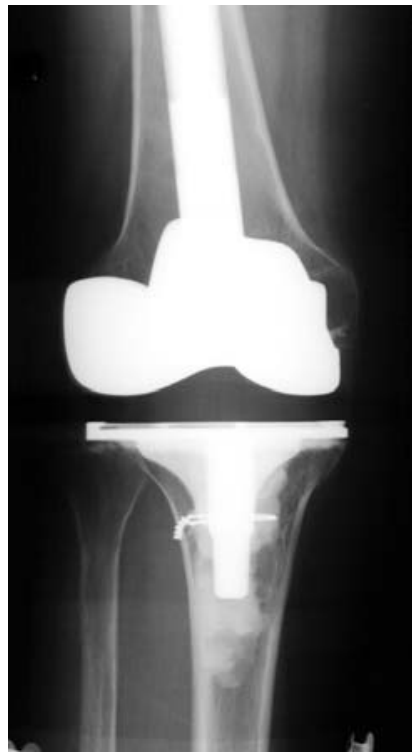

Fig. 1a

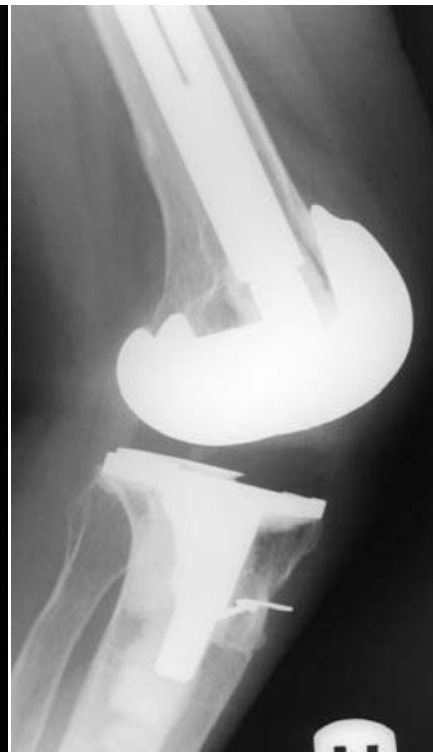

Fig. 1b

Radiographs 24 months after surgery. The calcaneal bone block of the allograft has incorporated into the proximal tibia.

native, scarred retinaculum and quadriceps. It was tightly sutured on both sides with the knee in full extension. The repair was then tested intra-operatively to demonstrate that the knee could be flexed to $50^{\circ}$ passively. The knee was immobilised post-operatively in a cast in full extension for six weeks. This was followed by a graduated physiotherapy programme similar to that described by Nazarian and Booth. ${ }^{8}$ The range of movement of the knee after three months was $70^{\circ}$, with full active extension and no extensor lag, and radiographs showed incorporation of the allograft bone block to the native tibia (Fig. 1). She developed reflex sympathetic dystrophy (RSD) six months post-operatively and investigations for infection were negative. The RSD was managed both physically and medically, albeit unsuccessfully for two years. However, she patient was satisfied with the range of movement of the knee, which improved to $100^{\circ}$ of flexion, with a $5^{\circ}$ extensor lag, at 18 months. By this time she had discontinued the use of a brace and had no symptoms of instability. She was reluctant to undergo any invasive spinal procedures in order to manage her RSD and chose further, non-operative management. By 24 months after surgery, she elected to undergo a right above-knee amputation because of severe pain secondary to RSD which was unresponsive to extensive conservative management. By this time the range of movement of the knee had decreased to $90^{\circ}$ of flexion, again with a $5^{\circ}$ extensor lag although she was able to walk with a stick. Despite the pain she remained satisfied with the range of movement, stability and function of the knee.

The extensor mechanism allograft was harvested after the amputation and was separately examined by two musculoskeletal pathologists (VLF, BMW).

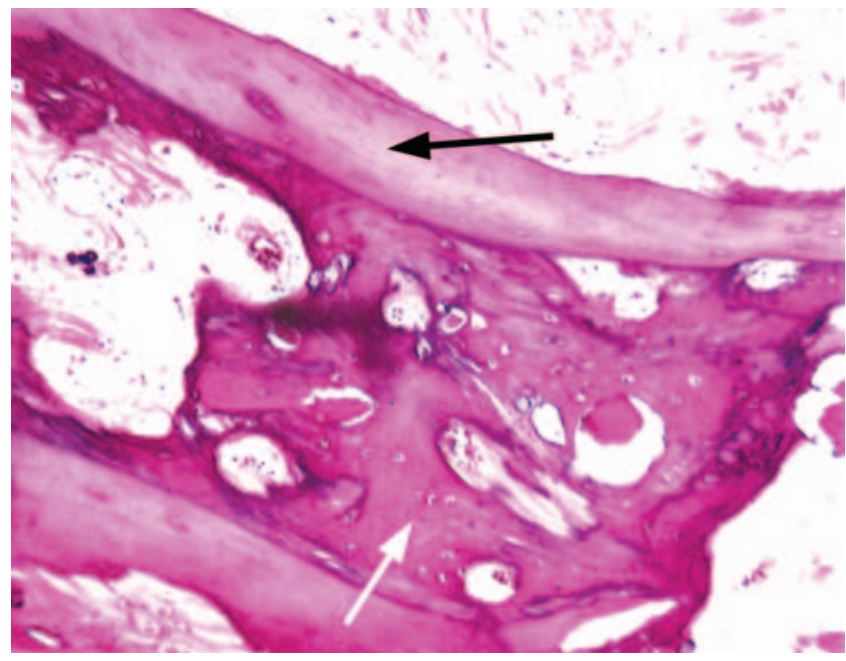

Fig. 2

The interface between allograft bone and host tibial bone shows incorporation. Newly-formed woven bone is in apposition to pre-existing trabeculae within the graft. The graft trabeculae are acellular (black arrow). The newly-formed bone is woven, with large rounded lacunae, each containing a small pinpoint nucleus (white arrow) (haematoxylin and eosin, $\times 10$ ).

\section{Histopathological results}

The allograft specimen was assessed in four regions: 1) the distal allograft calcaneal bone insertion into the host tibial bone; 2) the allograft tendon insertion site into the allograft calcaneal bone; 3 ) the mid-substance of the soft tissue allograft tendon at the level of the joint line and 4) the proximal junction of the allograft tendon with the host retinaculum and quadriceps. The purpose of the retrieval was to assess the incorporation of bone at the distal junction, the integrity of the allograft tendon insertion into the allograft bone, the integrity of the tendon in its mid-substance and of the proximal soft-tissue junction for degeneration, necrosis, inflammation, or immune reaction.

The interface between graft and host bone is seen in Figure 2. There was new, appositional, woven host bone penetrating into the original trabecular bone of the allograft, with an associated fibrous tissue ingrowth. The woven bone was in continuity with the graft, indicating maturation at the graft-host junction. These findings concurred with the radiographic incorporation of the allograft by host bone.

At the tendinous insertion of the allograft into calcaneal bone (Fig. 3), the gross specimen showed an intact insertion site. There was no histological evidence of necrosis or degeneration at this junction.

In the mid-substance of the allograft tendon the gross specimen showed signs of injury at the level of the superior surface of the polyethylene insert. A wedge of fibrinoid necrosis extended into the graft from posterior to anterior (Fig. 4). This may have represented a mechanical injury or degeneration of the graft. Five centimetres above the level of the joint line (Fig. 5), in the region of the anterior flange 


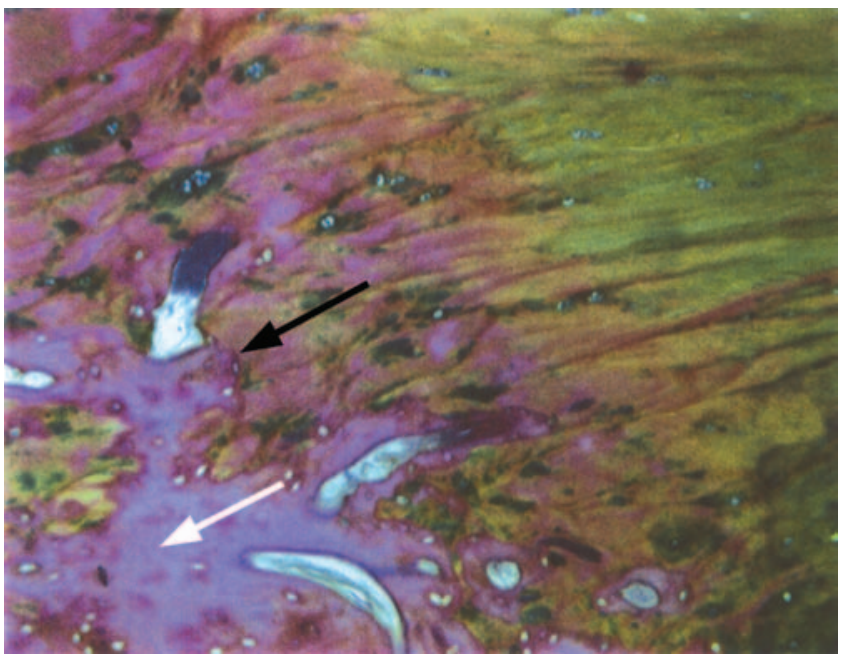

Fig. 3

Insertion of allograft tendo Achillis into the calcaneal bone block. The junction is well-preserved, without degeneration or necrosis. The recognisable tidewater mark (black arrow) extends diagonally from the top left to the bottom right corner. There is mucopolysaccharide preservation around the chondrocytes within the interface. Note the acellularity (white arrow) of the allograft bone at the bottom left corner (World Health Organization (WHO), x50 WHO stain: haematoxylin, saffron, alcian green and phloxin).

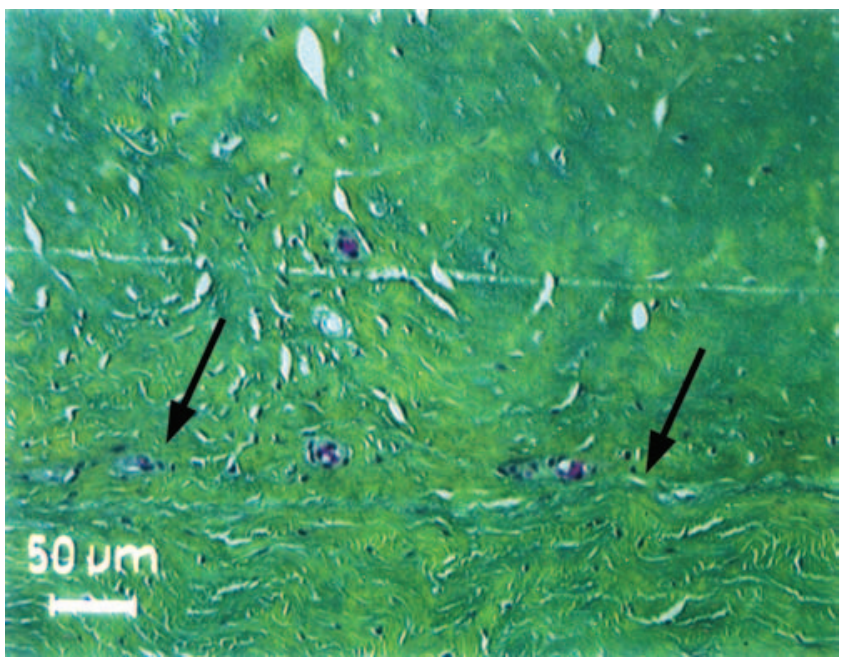

Fig. 5

Allograft tendon proximal to the knee joint at the level of the femoral component. The intact interface (black arrows) is seen between the allograft tendon (top) and the host tissue (bottom) in cross-section. The beginnings of vascularisation and population by host cells growing into the graft from the surrounding host tissue is seen. There is a row of blood vessels containing red blood cells at the interface and a single blood vessel containing red blood cells within the graft itself. A small number of nuclei are visible in the graft near the interface with the host tissue. The appearance of nuclei in allografts is regarded as an indicator of cell repopulation and viability. There is no degeneration or necrosis at this level (WHO section, x200).

of the femoral component, the allograft tendon was encased by dense fibrous host tissue with moderate vascularisation and preservation of the graft-host junction. There

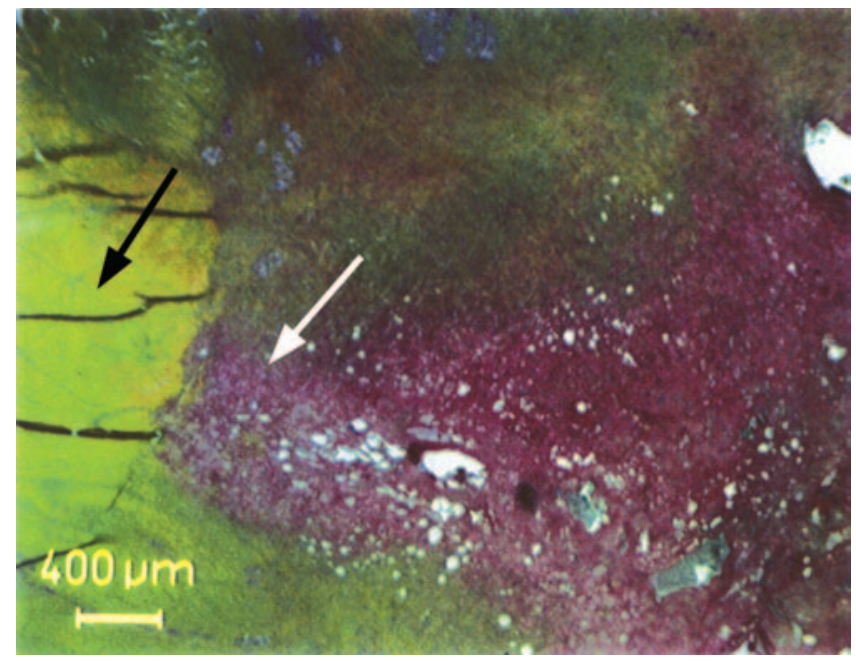

Fig. 4

Allograft tendon at the level of the prosthetic joint surface. On the left is the anterior (preserved) surface of the tendon and on the right is the interior (posterior) surface of the tendon adjacent to the prosthesis. A wedge of fibrinoid necrosis is present (white arrow) on the free surface of the graft. The intact graft is on the left (black arrow). Injury in this central region of the graft in a wedge-shaped pattern may be secondary to impingement from the prosthesis (WHO section, $\mathrm{x} 10$ ).

was no histological evidence of injury or necrosis at that level.

At the proximal junction of allograft tendon and host quadriceps tendon/retinaculum, the junction was grossly intact, without attenuation of the allograft. Histologically, the interface between the tissues was still evident (Figs 6 and 7). Dense host fibrous connective tissue encased the allograft tendon. There was no histological evidence of necrosis, inflammation, or collagen breakdown.

\section{Discussion}

To our knowledge, this is the first report of the retrieval of a well-functioning extensor mechanism allograft which had been used to reconstruct the knee extensor mechanism at TKA.

The use of an allograft under such circumstances has been reported before but with variable results. ${ }^{1,4,5,8,10}$ Emerson et $\mathrm{al}^{4}$ first described the technique (quadriceps tendon - patella - patellar tendon - tibial tubercle, with the patella resurfaced) but it was complicated by an extensor lag of $20^{\circ}$ to $40^{\circ}$ in one-third of their patients who required revision allograft procedures. Failure occurred from graft attenuation at the proximal anastomosis (host quadriceps allograft tendon junction) and patellar tendon ruptures occurred at its origin from the allograft patella, all within three months. There was satisfactory union at the tibial junction in all patients.

Nazarian and Booth ${ }^{8}$ modified this technique by tensioning the allograft tightly with the knee in full extension, permitting little or no intra-operative flexion. They reported a $22 \%$ rate of allograft rupture (eight of 36 patients), all of 


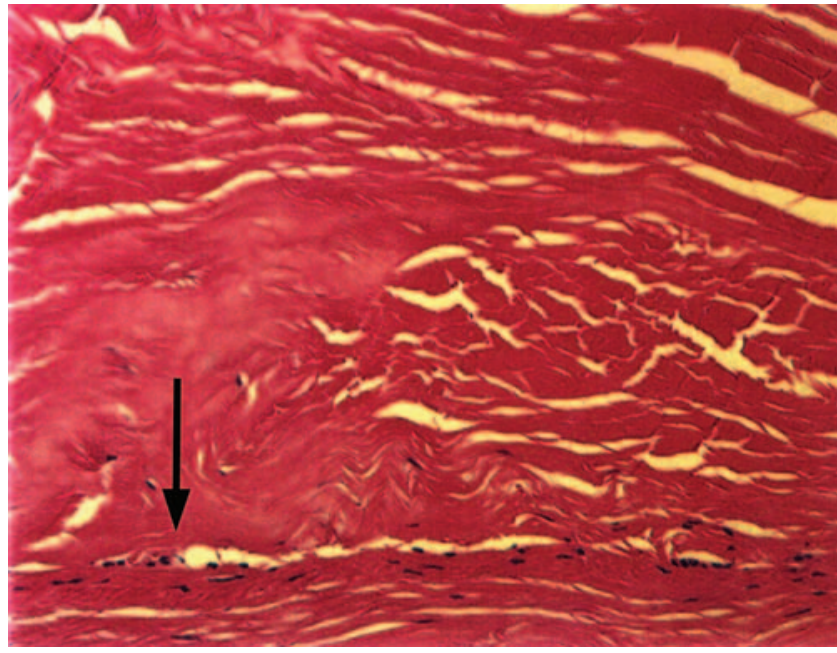

Fig. 6

The proximal junction between the allograft tendo Achilles and the host quadriceps tendon and retinaculum. The interface is denoted by a gap containing cells and blood vessels (black arrow). At the bottom of the image, there is host tissue with well-preserved cells and nuclei. The allograft ligament is in the upper two-thirds of the image and is acellular. There are occasional nuclei in the graft tendon immediately adjacent to the host tissue (haematoxylin and eosin, x100).

which required a revision with another allograft. The failures occurred between two months and four years postoperatively. Six occurred at the proximal junction, with attenuation and breakdown of the quadriceps anastomosis. The authors suggested that the use of a semi-constrained prosthesis in order to protect the allograft was important for a successful outcome.

Similarly, Leopold et $\mathrm{al}^{7}$ reported a $100 \%$ clinical failure rate (seven grafts), and a mean extensor lag of $59^{\circ}$, with the use of a knee-extensor mechanism allograft at a mean of 39 months after surgery. Attenuation and, less commonly, rupture of the graft occurred. The authors proposed that the mechanism of failure may involve attenuation of the allograft tissue rather than rupture at the sites of anastomosis.

Crossett et $\mathrm{al}^{10}$ used the same technique as ourselves on nine patients who required reconstruction of a deficient extensor mechanism at revision TKA. They reported two failures, both at the distal bone junction.

In our case the insertion of the distal bone block showed histological evidence of host bone incorporation into the allograft. Failures at this junction in previous reports ${ }^{7,10}$ have occurred early and typically involved avulsion of the allograft bone fragment. The necrosis in the central region at the level of the prosthetic joint line is an interesting and unique finding and may represent injury due to impingement of the implant on the graft, or degenerative necrosis secondary to attenuation in its mid-substance. Above the level of the joint line, the graft and host retinaculum remained in continuity, and without evidence of graft injury or necrosis of the allograft secondary to movement over the

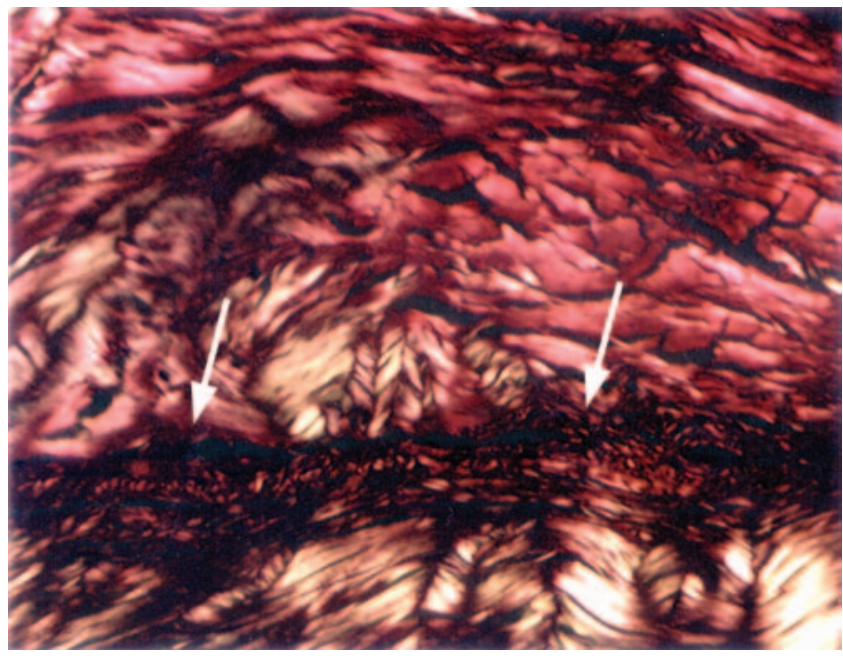

Fig. 7

Polarised light image of Figure 6 showing the similarity between the constituent collagen bundles of the host and graft tendons. Loose fibrous tis sue forms the non-birefringent interface layer between the two (white arrows). The collagen in the host tissue (lower half of the image) is welldefined and organised. The collagen in the graft tissue (upper half) appears smudgy and coalescent (polarized light, haematoxylin and eosin $\mathrm{x} 100$ ), indicating qualitative change in the allograft collagen over time.

flange of the femoral component. Our finding disagrees with the theory proposed by Nazarian and Booth ${ }^{8}$ who preferred the use of a quadriceps tendon-patella-patella tendon-bone graft as opposed to tendo-Achillis-bone allograft. They suggested that the latter might be susceptible to chronic attenuation of the graft over the anterior flange of the femoral component and that the patella may protect against this phenomenon. Our study demonstrated both grossly and histologically that the tendinous allograft proximal to the level of the joint line functioned well without compromise to the part which passed over the femoral flange.

The technique of preparation, handling, and insertion of the allograft in combination with implant stability and proper rotational alignment of the prosthetic components are all likely to play a role in the survival of an extensor mechanism allograft. Future long-term retrieval analyses of these allografts will allow further understanding of the success and of modes of failure of this technique.

No benefits in any form have been received or will be received from a commercial party related directly or indirectly to the subject of this article.

\section{References}

1. Lynch AF, Rorabeck CH, Bourne RB. Extensor mechanism complications following total knee arthroplasty. J Arthroplasty 1987;2:135-40.

2. Rand JA, Morrey BF, Bryan RS. Patellar tendon rupture after total knee arthroplasty. Clin Orthop 1989;244:233-8.

3. Barrack RL, Lyons T. Proximal tibia-extensor mechanism composite allograft for revision TKA with chronic patellar tendon rupture. Acta Orthop Scand 2000;71:419-21.

4. Emerson RH Jr, Head WC, Malinin TI. Reconstruction of patellar tendon rupture after total knee arthroplasty with an extensor mechanism allograft. Clin Orthop 1990;260:154-61. 
5. Emerson RH Jr, Head WC, Malinin TI. Extensor mechanism reconstruction with an allograft after total knee arthroplasty. Clin Orthop 1994;303:79-85.

6. Kulkarni S, Sawant M, Ireland J. Allograft reconstruction of the extensor mechanism for progressive extensor lag after total knee arthroplasty and previous patellectomy: a 3-year follow-up. J Arthroplasty 1999;14:892-4.

7. Leopold SS, Greidanus N, Paprosky WG, Berger RA, Rosenberg AG. High rate of failure of allograft reconstruction of the extensor mechanism after total knee arthroplasty. J Bone Joint Surg [Am] 1999;81-A:1574-9

8. Nazarian DG, Booth RE Jr. Extensor mechanism allografts in total knee arthroplasty. Clin Orthop 1999;367:123-9.

9. Zanotti RM, Freiberg AA, Matthews LS. Use of patellar allograft to reconstruct a patellar tendon-deficient knee after total joint arthroplasty. J Arthroplasty 1995;10:271-4.
10. Crossett LS, Sinha RK, Sechriest VF, Rubash JE. Reconstruction of a ruptured patellar tendon with achilles tendon allograft following total knee arthroplasty. J Bone Joint Surg [Am]2002;84-A:1354-61.

11. Cameron HU, Hu C. Results of total knee arthroplasty following takedown of formal knee fusion. J Arthroplasty 1996;11:732-7.

12. Henkel TR, Boldt JG, Drobny TK, Munzinger UK. Total knee arthroplasty after formal knee fusion using unconstrained and semiconstrained components: a report of 7 cases. J Arthroplasty 2001;16:768-76.

13. Kim YH, Kim JS, Cho SH. Total knee arthroplasty after spontaneous osseous ankylosis and takedown of formal knee fusion. J Arthroplasty 2000;15:453-60.

14. Naranja RJ Jr, Lotke PA, Pagnano MW, Hanssen AD. Total knee arthroplasty in a previously ankylosed or arthrodesed knee. Clin Orthop 1996:331:234-7. 